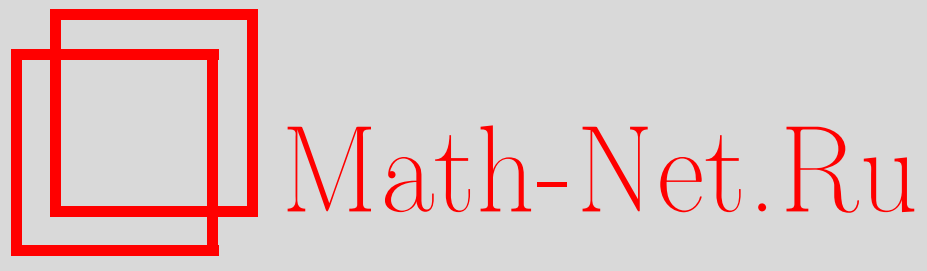

С. А. Локтев, Б. Л. Фейгин, О финитизации тождеств Гордона, Функи. анализ и его прил., 2001, том 35, выпуск 1, 53-61

DOI: https://doi.org/10.4213/faa231

Использование Общероссийского математического портала MathNet.Ru подразумевает, что вы прочитали и согласны с пользовательским соглашением

http://www . mathnet.ru/rus/agreement

Параметры загрузки :

IP : 35.174 .16 .151

26 апреля 2023 г., 10:34:06

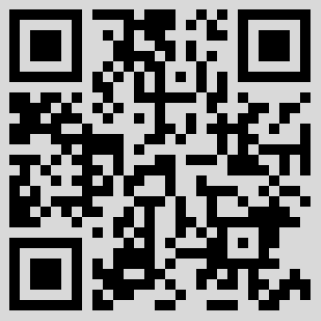


Функциональный анализ и его приложения

2001, т. 35, вып. 1, с. 53-61

УДК 512.81

\title{
О финитизации тождеств Гордона
}

\author{
(c) 2001. С. А. Локтев, Б. Л. ФЕйгин
}

\section{Введение}

Пусть $\widehat{S L_{2}}$ - группа, отвечающая аффинной алгебре Ли $\widehat{s l_{2}}$. Она является центральным расширением группы матриц размера $2 \times 2$ с определителем единица над кольцом функций на окружности. Символом $\widehat{\mathscr{N}}$ обозначим подгруппу группы $\widehat{S L_{2}}$, состоящую из верхнетреугольных матриц с единицами на диагонали, а символом $\widehat{n}$ обозначим алгебру Ли группы $\widehat{\mathscr{N}}$. Группа $\widehat{\mathscr{N}}$ состоит из токов на окружности со значениями в максимальной нильпотентной подгруппе группы $S L_{2}$. Алгебра $\widehat{n}$ абелева и может быть отождествлена с пространством функций на окружности. Выберем базис $\left\{e_{i}\right\}, i \in \mathbb{Z}$, в $\widehat{n}$, такой, что элементу $e_{m}$ соответствует функция $e^{2 \pi i m \phi}$, где $\phi-$ угловой параметр на окружности. Пусть $G r-$ аффинный грассманиан для группы $\widehat{S L_{2}}$, т. е. фактор группы $\widehat{S L_{2}}$ по максимальной параболической подгруппе $P$. Будем предполагать, что $e_{i}$ принадлежат алгебре Ли группы $P$ при $i \leqslant 0$. Обозначим через $p \in G r$ образ $P$ в многообразии $G r$.

В работе [2] изучается замыкание $\widehat{\mathscr{N}}$-орбиты точки $p$ на $G r$. Обозначим это многообразие через $\mathscr{M}$. Геометрическое определение многообразия $\mathscr{M}$ довольно сложно, более простым и прозрачным представляется описание $\mathscr{M}$ в терминах его координатного кольца.

Напомним, что на многообразии $G r$ существует линейное расслоение $\xi$, такое, что в сечениях расслоения $\xi^{\otimes k}$ реализуется неприводимое вакуумное представление $L_{0, k}$ уровня $k$ алгебры $\widehat{s l_{2}}$. Более точно, $H^{0}\left(G r, \xi^{\otimes k}\right) \cong\left(L_{0, k}\right)^{*}$. Таким образом, координатное кольцо многообразия $G r$ есть прямая сумма всех $\left(L_{0, k}\right)^{*}$ с естественным умножением $\left(L_{0, k_{1}}\right)^{*} \otimes\left(L_{0, k_{2}}\right)^{*} \rightarrow\left(L_{0, k_{1}+k_{2}}\right)^{*}$.

Многообразие $\mathscr{M}$ - подмногообразие многообразия $G r$, и, значит, его координатное кольцо есть фактор координатного кольца многообразия $G r$. А именно, пусть $v_{k}$ - старший вектор представления $L_{0, k}$ относительно подгруппы $P$. В $L_{0, k}$ имеется подпространство $D(k)=U(\widehat{n}) v_{k}$. Тогда координатное кольцо многообразия $\mathscr{M}$ есть прямая сумма пространств $D(k)^{*}$ с индуцированным умножением.

В интегрируемых представлениях алгебры $\widehat{s l_{2}}$ уровня $k$ выполняется соотношение $e(t)^{k+1}=0$, где $e(t)=\sum e_{i} t^{-i}$. Исходя из этого соотношения, в [2] строится следующая функциональная реализация пространств $D(k)^{*}$ :

$$
D(k)^{*}=\Sigma_{0}(k) \oplus \Sigma_{1}(k) \oplus \Sigma_{2}(k) \oplus \ldots,
$$

где $\Sigma_{m}(k)$ - пространство симметрических многочленов от $m$ переменных $t_{1}, \ldots, t_{m}$, делящихся на произведение $t_{1} \cdots t_{m}$ и обращающихся в нуль при $t_{1}=\cdots=t_{k+1}$. 
В этих терминах умножение на прямой сумме пространств $D(k)^{*}$ определяется как набор отображений $\Sigma_{m_{1}}\left(k_{1}\right) \otimes \Sigma_{m_{2}}\left(k_{2}\right) \rightarrow \Sigma_{m_{1}+m_{2}}\left(k_{1}+k_{2}\right)$. При этом образом пары полиномов $P_{1}\left(t_{1}, \ldots, t_{m_{1}}\right)$ и $P_{2}\left(t_{1}, \ldots, t_{m_{2}}\right)$ будет симметризация по переменным $t_{1}, \ldots, t_{m_{1}+m_{2}}$ их произведения:

$$
\left(P_{1} \circ P_{2}\right)\left(t_{1}, \ldots, t_{m_{1}+m_{2}}\right)=\operatorname{Symm}\left(P_{1}\left(t_{1}, \ldots, t_{m_{1}}\right) P_{2}\left(t_{m_{1}+1}, \ldots, t_{m_{1}+m_{2}}\right)\right) .
$$

Пусть $N$ - натуральное число. Пусть $\widehat{n}_{N}$ - подалгебра алгебры $\widehat{n}$, порожденная $e_{i}$ при $i>N$, и пусть $\widehat{\mathscr{N}}_{N}-$ соответствующая подгруппа. Определим многообразие $\mathscr{M}_{N}$ как фактор многообразия $\mathscr{M}$ по группе $\widehat{\mathscr{N}}_{N}$. С геометрической точки зрения для этого необходимо удалить из многообразия $\mathscr{M}$ нестабильные орбиты, а остаток профакторизовать по действию группы. С алгебраической точки зрения координатное кольцо многообразия $\mathscr{M}_{N}$ будет подкольцом $\widehat{\mathscr{N}}_{N}$-инвариантов (или, что то же самое, $\widehat{n}_{N}$-инвариантов) в координатном кольце многообразия $\mathscr{M}$.

Таким образом, координатным кольцом многообразия $\mathscr{M}_{N}$ будет прямая сумма пространств $D_{N}(k)^{*}$, двойственных к пространствам $D_{N}(k)=\widehat{n}_{N} \backslash D(k)$ коинвариантов пространства $D(k)$ относительно алгебры $\widehat{n}_{N}$. Умножение на этом пространстве определяется тем же самым способом. Теперь определим многообразие $\mathscr{M}_{N}$ как проективный спектр градуированной алгебры $\bigoplus D_{N}(k)^{*}$. Автоматически при этом получим линейное расслоение $\xi_{N}$ на $\mathscr{M}_{N}$, такое, что $H_{0}\left(\mathscr{M}_{N}, \xi_{N}^{\otimes k}\right) \cong D_{N}(k)^{*}$.

На многообразии $G r$ действует двумерный тор $T$, касательное пространство которого в единице порождено картановским элементом $h_{0}$ и элементом энергии $L_{0}$. Легко проверить, что это действие продолжается до действия тора $T$ на многообразиях $\mathscr{M}$ и $\mathscr{M}_{N}$; при этом действие тора $T$ на $D(k)$ и $D_{N}(k)$ определяется тем, что $\left[h_{0} / 2, e_{i}\right]=e_{i},\left[L_{0}, e_{i}\right]=i e_{i}$.

Пусть

$$
d_{N}(k ; q, z)=\operatorname{Tr}_{D_{N}(k)} q^{L_{0}} z^{h_{0} / 2}
$$

- полином Гильберта градуированного пространства $D_{N}(k)$. Вычислить этот полином можно двумя способами, как это было проделано для пространств $D(k)$ в [2]. Первый из них - чисто алгебраический. Он основан на функциональной интерпретации пространства $D_{N}(k)^{*}$ и фильтрации Гордона на пространстве симметрических полиномов. Соответствующее вычисление проделано в [1], и его результат мы приводим в левой части формулы (2) ниже. Второй способ - применение голоморфной формулы Лефшеца к расслоению $\xi_{N}^{\otimes k}$. Остановимся на нем подробнее.

Можно доказать, что при четном $N$ многообразие $\mathscr{M}_{N}$ неособо (а при нечетном $N>3$ содержит единственную особую точку) и $H^{i}\left(\mathscr{M}_{N}, \xi_{N}^{\otimes k}\right)=0$ для $i>0$. При этом неподвижные точки действия тора $T$ на $\mathscr{M}_{N}$ нумеруются парой неотрицательных чисел $m$ и $n$, таких, что $m+n \leqslant N / 2$. Неподвижная точка $x_{0,0}-$ образ выделенной точки $p \in G r$ в $\mathscr{M}_{N}$. Положим для $t \in \mathbb{R}$

$$
x_{m, n}(t)=\prod_{i=0}^{m-1} \exp \left(t e_{2 i+1}\right) \prod_{i=0}^{n-1} \exp \left(t e_{N-2 i}\right) \cdot x_{0,0} .
$$

Тогда неподвижной точкой $x_{m, n}$ будет предел $\lim _{t \rightarrow \infty} x_{m, n}(t)$. Учитывая вклады этих точек, получим правую часть формулы (2). Это выражение также приведено в [6]. 
Таким образом, для четного $N$ получаем формулу

$$
\begin{aligned}
& \sum_{v_{1}, \ldots, v_{k} \in \mathbb{Z}_{+}} q^{\sum_{i, j} v_{i} v_{j} \min (i, j)} z^{\sum_{i} i v_{i}} \prod_{i=1}^{k}\left[\begin{array}{c}
(N+1) i-2 \sum_{j} v_{j} \min (i, j)+v_{i} \\
v_{i}
\end{array}\right]_{q} \\
= & \sum_{\substack{m, n \in \mathbb{Z}_{+} \\
m+n \leqslant N / 2}} \frac{z^{k(m+n)} q^{k\left(m^{2}+(N+1) n-n^{2}\right)}}{\prod_{i=1}^{m}\left(1-q^{i}\right)\left(1-q^{-i-m+1} z^{-1}\right) \prod_{i=2 m+1}^{N-2 n}\left(1-q^{i} z\right) \prod_{i=1}^{n}\left(1-q^{-i}\right)\left(1-q^{-N+i+n-2} z^{-1}\right)},
\end{aligned}
$$

где $\left[\begin{array}{c}n \\ m\end{array}\right]_{q}$ есть $q$-биномиальный коэффициент, а выражение $1 /\left(1-q^{\alpha} z^{\beta}\right)$ следует понимать как $\sum_{i \geqslant 0} q^{i \alpha} z^{i \beta}$ при $\alpha, \beta \geqslant 0$ и как $-\sum_{i<0} q^{i \alpha} z^{i \beta}$ при $\alpha, \beta \leqslant 0$.

Правая часть этой формулы похожа на знакопеременную сумму в формуле Вейля. Напомним, что формулу Вейля для характеров интегрируемых представлений алгебры Каца-Муди можно интерпретировать геометрически как формулу Лефшеца для расслоений на соответствующем многообразии флагов. Неподвижные точки действия тора на многообразии флагов нумеруются элементами группы Вейля и, таким образом, соответствуют экстремальным векторам рассматриваемого представления. При этом вклад неподвижной точки в формулу Лефшеца можно интерпретировать как характер представления «в окрестности» соответствующего экстремального вектора. Поясним это для представлений группы $\widehat{S L_{2}}$.

Пусть $L_{l, k-l}$ - интегрируемое представление группы $\widehat{S L_{2}}$ и $v_{k, l}$ - его старший вектор. Рассмотрим экстремальный вектор $w\left(v_{k, l}\right)$, где $w-$ элемент группы Вейля группы $\widehat{S L_{2}}$. Пусть $c_{q}(w ; k, l), c_{z}(w ; k, l)$ - градуировки этого вектора. Будем называть предел

$$
q^{c_{q}(w ; k, l)} z^{c_{z}(w ; k, l)} \lim _{r \rightarrow \infty}\left(q^{-c_{q}(w ; r k, r l)} z^{-c_{z}(w ; r k, r l)} \operatorname{ch} L_{r l, r(k-l)}\right)
$$

характером представления $L_{l, k-l}$ «в окрестности» вектора $w\left(v_{k, l}\right)$. Легко проверить, что это характер модуля Верма $M_{l, k-l}$, к которому применен элемент $w$. Чтобы получить вклад вектора $w\left(v_{k, l}\right)$, необходимо разложить выражение $(3)$ по положительным степеням $q$. При этом получится характер некоторого модуля Верма со знаком, равным $(-1)^{l(w)}$. Формула Вейля утверждает, что характер представления $L_{l, k-l}$ будет равен сумме таких вкладов.

Мы применим эти соображения для вычисления характеров пространств $D_{N}(k)$. А именно, мы представим характер пространства $D_{N}(k)$ в виде суммы характеров «в окрестности» некоторых векторов. В результате мы получим обобщение формулы (2), которое докажем комбинаторными методами. На самом деле знакопеременная часть этой формулы также будет формулой Лефшеца для расслоений на некотором многообразии.

1. Рассмотрим пространство

$$
D_{N}(k, l, r)=\mathbb{C}\left[e_{1}, \ldots, e_{N}\right] / I_{N}(k, l, r), \quad N \geqslant 2,
$$

где идеал $I_{N}(k, l, r)$ порожден элементами

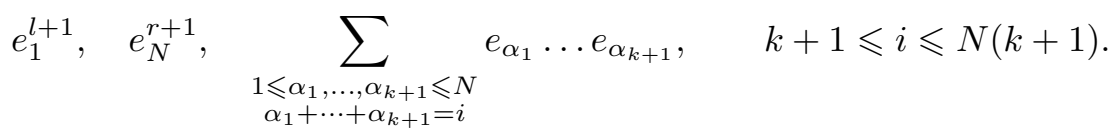


Другими словами, $I_{N}(k, l, r)$ порожден элементами $e_{1}^{l+1}, e_{N}^{r+1}, e(t)^{k+1}, t \in \mathbb{C}$, где $e(t)=\sum_{i=1}^{N} e_{i} t^{i}$.

В частности, пространство $D_{N}(k)$, обсуждаемое во введении, изоморфно $D_{N}(k, k, k)$.

Введем на пространстве $D_{N}(k, l, r)$ биградуировку $\left(\operatorname{deg}_{q}, \operatorname{deg}_{z}\right)$. Для этого биградуируем алгебру $\mathbb{C}\left[e_{1}, \ldots, e_{N}\right]$, полагая $\operatorname{deg}_{q} e_{i}=i, \operatorname{deg}_{z} e_{i}=1$. Поскольку элементы из $I_{N}(k, l, r)$ однородны, биградуировка алгебры $\mathbb{C}\left[e_{1}, \ldots, e_{N}\right]$ определяет биградуировку на $D_{N}(k, l, r)$. Символом $d_{N}(k, l, r ; q, z)$ обозначим полином Гильберта этого пространства:

$$
d_{N}(k, l, r ; q, z)=\sum_{i, j \geqslant 0} q^{i} z^{j} \operatorname{dim}\left(D_{N}(k, l, r)\right)^{i, j} .
$$

2. Пространство, двойственное к $D_{N}(k, l, r)$, допускает удобную функциональную реализацию, которую мы сейчас опишем.

Пусть $\Sigma_{m}^{N}-$ пространство симметрических полиномов вида

$$
t_{1} \cdots t_{m} f\left(t_{1}, \ldots, t_{m}\right)
$$

степени не выше $N$ по каждой из $m$ переменных. Обозначим через $\Sigma_{m}^{N}(k, l, r)$ подпространство в $\Sigma_{m}^{N}$, состоящее из полиномов вида (4), таких, что

1) $f\left(t_{1}, \ldots, t_{m}\right)=0$, если $t_{1}=\cdots=t_{k+1}$,

2) $f\left(t_{1}, \ldots, t_{m}\right)=0$, если $t_{1}=\cdots=t_{l+1}=0$,

3) многочлен $f\left(t, \ldots, t, t_{r+2}, t_{r+3}, \ldots, t_{m}\right)$ имеет степень, строго меньшую $(r+1)(N-1)$, по переменной $t$.

ПреДЛОЖенИЕ 1. Пространство $D_{N}(k, l, r)$ двойственно прямой сумме

$$
\Sigma_{0}^{N}(k, l, r) \oplus \Sigma_{1}^{N}(k, l, r) \oplus \Sigma_{2}^{N}(k, l, r) \oplus \ldots .
$$

При этом подпространство $\left(D_{N}(k, l, r)\right)^{i, j}$ двойственно подпространству в $\Sigma_{j}^{N}(k, l, r)$, состоящему из полиномов степени $i$.

Двойственность строится следующим образом. Пусть $S_{N}^{m}-$ пространство однородных полиномов степени $m$ от $N$ переменных $e_{1}, \ldots, e_{N}$. Определим отображение $\mathscr{P}:\left(S_{N}^{m}\right)^{*} \rightarrow \Sigma_{m}^{N}$, полагая для $\phi \in\left(S_{N}^{m}\right)^{*}$

$$
\mathscr{P}(\phi)\left(t_{1}, \ldots, t_{m}\right)=\phi\left(e\left(t_{1}\right) \cdots e\left(t_{m}\right)\right) .
$$

Для доказательства предложения теперь достаточно заметить, что это отображение - изоморфизм, а также то, что условие 1) на подпространство $\Sigma_{m}^{N}(k, l, r)$ соответствует факторизации двойственного пространства по $e(t)^{k+1}$, условие 2$)-$ по $e_{1}^{l+1}$, условие 3$)-$ по $e_{N}^{r+1}$.

3. Заметим, что на пространстве $D_{N}^{\vee}=\bigoplus_{m, k, l, r} \Sigma_{m}^{N}(k, l, r)$ определено ассоциативное коммутативное умножение. Произведение полиномов $P_{1} \in \Sigma_{m_{1}}^{N}\left(k_{1}, l_{1}, r_{1}\right)$ и $P_{2} \in \Sigma_{m_{2}}^{N}\left(k_{2}, l_{2}, r_{2}\right)$ определим по формуле (1). Ясно, что $P_{1} \circ P_{2} \in \Sigma_{m_{1}+m_{2}}^{N}\left(k_{1}+k_{2}\right.$, $\left.l_{1}+l_{2}, r_{1}+r_{2}\right)$.

Наличие структуры коммутативной алгебры на пространстве $D_{N}^{\vee}$ приводит к следующему утверждению.

ПредЛожение 2. Существует многообразие $\mathscr{M}_{N}^{\prime}$, а также линейные расслоения $\xi_{K}, \xi_{L}, \xi_{R}$ и векторные поля $L_{q}, L_{z}$ на $\mathscr{M}_{N}^{\prime}$, такие, ито $D_{N}(k, l, r)$ 
двойственно $H^{0}\left(\mathscr{M}_{N}^{\prime}, \xi_{K}^{\otimes k} \otimes \xi_{L}^{\otimes l} \otimes \xi_{R}^{\otimes r}\right) u d_{N}(k, l, r ; q, z)$ - след действия оператора $q^{L_{q}} z^{L_{z}}$ на этом пространстве.

Многообразие $\mathscr{M}_{N}^{\prime}$ строится следующим образом. Пусть $S$ - спектр алгебры $D_{N}^{\vee}$. Алгебра $D_{N}^{\vee}=\bigoplus D_{N}(k, l, r)^{*}$ триградуирована числами $k, l$ и $r$, что определяет действие трехмерного комплексного тора $T_{3}$ на $S$. Пусть $\widetilde{\mathscr{M}}_{N}^{\prime}-$ максимальное подмногообразие в $S$, на котором тор $T_{3}$ действует свободно. Положим $\mathscr{M}_{N}^{\prime}=\widetilde{\mathscr{M}}_{N}^{\prime} / T_{3}$. Тогда в качестве расслоений $\xi_{K}, \xi_{L}, \xi_{R}$ можно взять расслоения, ассоциированные с соответствующими одномерными представлениями тора $T_{3}$.

4. Фильтрация Гордона на пространствах $\Sigma_{m}^{N}$ (см. $\left.[1,2]\right)$ позволяет получить формулу, обобщающую левую часть формулы (2). А именно:

Теорема 1. Пусть $Q-$ матрица размера $k \times k$, такая, что $Q_{i, j}=\min (i, j)$; пусть $\mathscr{L}$ и $\mathscr{R}-$ векторьь в $\mathbb{R}^{k}$, такие, ито

$$
\mathscr{L}_{i}=\left\{\begin{array}{ll}
i-l, & i \geqslant l, \\
0, & i \leqslant l,
\end{array} \quad \mathscr{R}_{i}= \begin{cases}i-r, & i \geqslant r, \\
0, & i \leqslant r .\end{cases}\right.
$$

Тогда

$$
d_{N}(k, l, r ; q, z)=\sum_{\substack{v=\left(v_{1}, \ldots, v_{k}\right) \\
v_{i} \in \mathbb{Z}_{+}}} q^{v^{t} Q v+\mathscr{L}^{t} v} z^{\sum i v_{i}} \prod_{i=1}^{k}\left[\begin{array}{c}
(N+1) i-(2 Q v+\mathscr{L}+\mathscr{R}-v)_{i} \\
v_{i}
\end{array}\right]_{q},
$$

где значок ${ }^{t}$ превращает вектор-столбец в вектор-строку, а

$$
\left[\begin{array}{c}
n \\
m
\end{array}\right]_{q}=\frac{\prod_{i=1}^{n}\left(1-q^{i}\right)}{\prod_{i=1}^{m}\left(1-q^{i}\right) \prod_{i=1}^{n-m}\left(1-q^{i}\right)} .
$$

Доказательство этой формулы повторяет доказательства аналогичных формул в [1] и [2].

5. Далее построим базис в пространстве $D_{N}(k, l, r)$.

Teоpema 2. Moномы $e_{1}^{a_{1}} \cdots e_{N}^{a_{N}}$, такие, umo

$$
a_{1} \leqslant l, \quad a_{N} \leqslant r, \quad a_{i}+a_{i+1} \leqslant k \quad \text { для всех } 1 \leqslant i<N,
$$

образуют базис в $D_{N}(k, l, r)$.

ДокАЗАТЕЛЬСтво. В [1] это утверждение доказано для $r=k$ и, поскольку $D_{N}(k, l, 0) \cong D_{N-1}(k, l, k)$, для $r=0$.

Прежде всего, из того, что мономы $e_{1}^{a_{1}} \cdots e_{N}^{a_{N}}$, удовлетворяющие условию $a_{i}+a_{i+1} \leqslant k$, порождают пространство $D_{N}(k, k, k)$, следует, что мономы, удовлетворяющим условиям $(5)$, порождают $D_{N}(k, l, r)$. Таким образом, для доказательства теоремы достаточно сравнить размерность пространства $D_{N}(k, l, r)$ и количество элементов предполагаемого мономиального базиса.

Обозначим через $\widetilde{D}_{N}(k, l, r)$ множество мономов, удовлетворяющих условиям (5). Кроме того, пусть $d_{N}(k, l, r)=\operatorname{dim} D_{N}(k, l, r)$, а $\tilde{d}_{N}(k, l, r)-$ количество элементов в $\widetilde{D}_{N}(k, l, r)$. Имеет место

ЛЕмма 1. (i) $\tilde{d}_{N}(k, l, r)=\tilde{d}_{N}(k, l, r-1)+\tilde{d}_{N-1}(k, l, k-r)$.

(ii) $d_{N}(k, l, r) \leqslant d_{N}(k, l, r-1)+d_{N-1}(k, l, k-r)$. 
ДокАЗАТЕЛЬСтво. (i) Пусть $m=e_{1}^{a_{1}} \cdots e_{N}^{a_{N}} \in \widetilde{D}_{N}(k, l, r)$. Тогда либо $a_{N}<r$ и $m \in \widetilde{D}_{N}(k, l, r-1)$, либо $a_{N}=r$ и $m^{\prime}=e_{1}^{a_{1}} \cdots e_{N-1}^{a_{N-1}} \in \widetilde{D}_{N-1}(k, l, k-r)$. И наоборот, по любому элементу из $\widetilde{D}_{N}(k, l, r-1)$ или из $\widetilde{D}_{N-1}(k, l, k-r)$ восстанавливается соответствующий элемент из $\widetilde{D}_{N}(k, l, r)$.

(ii) Рассмотрим отображение $\tilde{i}_{N}(r): \mathbb{C}\left[e_{1}, \ldots, e_{N-1}\right] \rightarrow D_{N}(k, l, r)$, такое, что $\tilde{i}_{N}(r)(P)=P \cdot e_{N}^{r}$. Легко проверить, что образ идеала $I_{N-1}(k, l, k-r)$ при этом отображении нулевой; таким образом, $\tilde{i}_{N}(r)$ индуцирует отображение $i_{N}(r)$ : $D_{N-1}(k, l, k-r) \rightarrow D_{N}(k, l, r)$. В частности, получаем, что $\operatorname{dim} \operatorname{Im}\left(i_{N}(r)\right) \leqslant$ $d_{N-1}(k, l, k-r)$.

Также легко проверить, что образ идеала $I_{N}(k, l, r-1)$ при проекции $D_{N}(k, l, r)$ $\rightarrow \operatorname{Coker}\left(i_{N}(r)\right)$ нулевой, а значит, $\operatorname{dim} \operatorname{Coker}\left(i_{N}(r)\right) \leqslant d_{N}(k, l, r-1)$. А поскольку $\operatorname{dim} D_{N}(k, l, r)=\operatorname{dim} \operatorname{Im}\left(i_{N}(r)\right)+\operatorname{dim} \operatorname{Coker}\left(i_{N}(r)\right)$, верно утверждение леммы.

Из равенства $d_{N}(k, l, r)=\tilde{d}_{N}(k, l, r)$ для $r=0, k$, а также для $N=2$ следует, что неравенство в утверждении (ii) леммы 1 является равенством. Тем самым получаем равенство $d_{N}(k, l, r)=\tilde{d}_{N}(k, l, r)$ для всех $r$ и утверждение теоремы 2.

СлЕДСтвие $1 . d_{N}(k, l, r ; q, z)=d_{N}(k, l, r-1 ; q, z)+\left(q^{N} z\right)^{r} d_{N-1}(k, l, k-r ; q, z)$.

6. Другое следствие из теоремы 2 можно сформулировать так. Пусть $P_{N}(k, l, r)-$ многогранник в $\mathbb{R}^{N}=\left\{\left(x_{1}, \ldots, x_{N}\right)\right\}$, заданный условиями

$$
x_{1} \leqslant l, \quad x_{N} \leqslant r, \quad x_{i}+x_{i+1} \leqslant k \text { для всех } 1 \leqslant i<N .
$$

Пусть $\varphi_{z}=\sum x_{i}, \varphi_{q}=\sum i x_{i}-$ функционалы на $\mathbb{R}^{N}$.

Следствие 2. Размерность пространства $D_{N}(k, l, r)$ совпадает с количеством целых точек многогранника $P_{N}(k, l, r)$, a

$$
d_{N}(k, l, r ; q, z)=\sum_{\mathbf{x} \in \mathbb{Z}^{N} \cap P_{N}(k, l, r)} q^{\varphi_{q}(\mathbf{x})} z^{\varphi_{z}(\mathbf{x})} .
$$

Следуя идеям, изложенным в [4], представим полином $d_{N}(k, l, r ; q, z)$ как сумму вкладов вершин многогранника $P_{N}(k, l, r)$. А именно, мы предъявим (см. теорему 3 ) формулу вида $d_{N}(k, l, r ; q, z)=\sum d_{M}$, где суммирование ведется по всем вершинам многогранника $P_{N}(k, l, r)$.

Пусть $M-$ некоторая вершина. В ее малой окрестности наш многогранник выглядит как конус над некоторым $(N-1)$-мерным многогранником. Обозначим этот конус через $C(M)$. В нашем случае для вершины $M$

$$
d_{M}=\sum_{\mathbf{x} \in \mathbb{Z}^{N} \cap C(M)} q^{\varphi_{q}(\mathbf{x})} z^{\varphi_{z}(\mathbf{x})} .
$$

В соответствии с [4] будем говорить, что вершина $M$ простая, если $C(M)-$ конус над симплексом. В этом случае получается простое выражение для вклада. Легко видеть, что при общих $k, l, r$ (при $k>l>0, k>r>0, l \neq r, l+r \neq k$ ) простые вершины многогранника $P_{N}(k, l, r)$ суть

$$
M_{m, n}=(\underbrace{l, k-l, l, k-l, \ldots}_{m}, \underbrace{0, \ldots, 0}_{N-m-n}, \underbrace{\ldots, r, k-r, r}_{n}) .
$$


При этом $n+m \leqslant N$, а в случае $n+m=N$ дополнительно требуется, чтобы сумма $m$-й и $(m+1)$-й координат не превышала $k$.

ПредлОЖЕНИЕ 3. Пусть $k, l$ u $r$ общие. Тогда всякая ц̧елая точка конуса $C\left(M_{m, n}\right)$ получается из $M_{m, n}$ добавлением линейной комбинации с цельми неотрицательными коэффицииентами векторов $v^{1}, \ldots, v^{N}$, где

$$
v^{i}= \begin{cases}(\underbrace{0, \ldots, 0}_{m-i}, \underbrace{-1,1, \ldots,(-1)^{i-1},(-1)^{i}}_{i}, \underbrace{0, \ldots, 0}_{N-m}), & i \leqslant m, \\ (\underbrace{0, \ldots, 0}_{i-1}, 1, \underbrace{0, \ldots, 0}_{N-i}), & m<i \leqslant N-n, \\ (\underbrace{0, \ldots, 0}_{N-n}, \underbrace{(-1)^{N-i+1},(-1)^{N-i}, \ldots, 1,-1}_{N-i+1}, \underbrace{0, \ldots, 0}_{n+i-N-1}), & N-n<i .\end{cases}
$$

Таким образом,

$$
d_{M_{m, n}}=q^{\varphi_{q}\left(M_{m, n}\right)} z^{\varphi_{z}\left(M_{m, n}\right)} \prod_{i=1}^{N} \frac{1}{1-q^{\varphi_{q}\left(v^{i}\right)} z^{\varphi_{z}\left(v^{i}\right)}} .
$$

Величины $\varphi_{q}\left(M_{m, n}\right)$ и $\varphi_{z}\left(M_{m, n}\right)$ являются линейными функциями от $k, l, r$. Величины $\varphi_{q}\left(v^{i}\right)$ и $\varphi_{z}\left(v^{i}\right)$ не зависят от $k, l, r$ и равны следующим числам:

\begin{tabular}{|c|c|c|c|c|c|}
\hline & \multicolumn{2}{|c|}{$m \geqslant i$} & \multirow{2}{*}{$N-n \geqslant i}$, & \multicolumn{2}{|c|}{$i>N-n$} \\
\cline { 6 - 6 } \cline { 5 - 6 } & $i$ четно & $i$ нечетно & $i>m$ & $N-i$ четно & $N-i$ нечетно \\
\hline$\varphi_{z}\left(v^{i}\right)$ & 0 & -1 & 1 & -1 & 0 \\
\hline$\varphi_{q}\left(v^{i}\right)$ & $i / 2$ & $(i-1) / 2-m$ & $i$ & $n-1-(3 N-i) / 2$ & $-(N-i+1) / 2$ \\
\hline
\end{tabular}

Таким образом,

$$
d_{M_{m, n}}=\frac{q^{\varphi_{q}\left(M_{m, n}\right)} z^{\varphi_{z}\left(M_{m, n}\right)}}{\prod_{i=1}^{\left[\frac{m}{2}\right]}\left(1-q^{i}\right) \prod_{i=\left[\frac{m}{2}\right]+1}^{m}\left(1-q^{-i} z^{-1}\right) \prod_{i=m+1}^{N-n}\left(1-q^{i} z\right) \prod_{i=1}^{\left[\frac{n}{2}\right]}\left(1-q^{-i}\right) \prod_{i=\left[\frac{n}{2}\right]+1}^{n}\left(1-q^{i-N-1} z^{-1}\right)} .
$$

Чтобы придать смысл этому выражению как ряду от положительных степеней $q$ и $z$, положим выражение $1 /\left(1-q^{\alpha} z^{\beta}\right)$ равным $\sum_{i \geqslant 0} q^{i \alpha} z^{i \beta}$ при $\alpha, \beta \geqslant 0$ и равным $-\sum_{i<0} q^{i \alpha} z^{i \beta}$ при $\alpha, \beta \leqslant 0$ (других случаев не возникает). Обозначим получившийся ряд через $d_{N}^{m, n}(k, l, r ; q, z)$. Формула (6) определяет его при любых целых $k, l, r$.

7. Эти соображения позволяют нам обобщить правую часть формулы (2). Оказывается, что в нашем случае достаточно сложить вклады простых вершин $M_{m, n}$, т. е. суммарный вклад остальных вершин нулевой. Положим

$$
\begin{aligned}
& d_{N}^{e}(k, l, r ; q, z)=\sum_{\substack{m+n<N \text { или } \\
m+n=N, m \text { четно }}} d_{N}^{m, n}(k, l, r ; q, z), \\
& d_{N}^{o}(k, l, r ; q, z)=\sum_{\substack{m+n<N \text { или } \\
m+n=N, m \text { нечетно }}} d_{N}^{m, n}(k, l, r ; q, z) .
\end{aligned}
$$

Теорема 3. (i) Если $N$ нечетно, $l \leqslant r, \operatorname{mo~}_{N}(k, l, r ; q, z)=d_{N}^{o}(k, l, r ; q, z)$.

(ii) Eсли $N$ нечетно, $l \geqslant r$, то $d_{N}(k, l, r ; q, z)=d_{N}^{e}(k, l, r ; q, z)$.

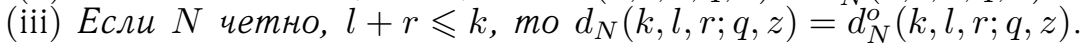

(iv) Eсли $N$ четно, $l+r \geqslant k$, mо $d_{N}(k, l, r ; q, z)=d_{N}^{e}(k, l, r ; q, z)$. 
ДокАЗАТЕЛЬСтво. Докажем несколько вспомогательных утверждений.

ЛЕмма 2. (i) $d_{N}^{e}(k, l, r ; q, z)=d_{N}^{e}(k, l, r-1 ; q, z)+\left(q^{N} z\right)^{r} d_{N-1}^{e}(k, l, k-r ; q, z)$.

(ii) $d_{N}^{o}(k, l, r ; q, z)=d_{N}^{o}(k, l, r-1 ; q, z)+\left(q^{N} z\right)^{r} d_{N-1}^{o}(k, l, k-r ; q, z)$.

ДокАЗАТЕЛЬСтво. Утверждения следуют из того, что

$$
d_{N}^{m, n}(k, l, r ; q, z)=d_{N}^{m, n}(k, l, r-1 ; q, z)+\left(q^{N} z\right)^{r} d_{N-1}^{m, n-1}(k, l, k-r ; q, z)
$$

при $n>0$ и $d_{N}^{m, 0}(k, l, r ; q, z)=d_{N}^{m, 0}(k, l, r-1 ; q, z)$.

Лемма 3. $d_{N}^{e}(k, l,-1 ; q, z)=0$ для нечетного $N$, a $d_{N}^{o}(k, l,-1 ; q, z)=0$ для четного $N$.

ДокАЗАТЕЛьство. Лемма следует из равенства

$$
d_{N}^{m, 2 n}(k, l,-1 ; q, z)=-d_{N}^{m, 2 n+1}(k, l,-1 ; q, z) .
$$

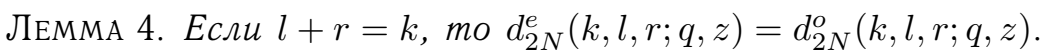

ДокАЗАТЕЛЬСтво. Нам надо доказать, что

$$
\sum_{m=0}^{2 N}(-1)^{m} d_{2 N}^{m, 2 N-m}(k, a, k-a ; q, z)=0
$$

при всех $k$ и $a$. Пусть

$$
\begin{gathered}
\Phi(n)=\prod_{i=1}^{n} \frac{1}{1-q^{i}}, \quad \Psi(m, n)=\prod_{i=m}^{n} \frac{1}{1-q^{-i} z^{-1}}, \quad \Phi^{\vee}(n)=\prod_{i=1}^{n} \frac{1}{1-q^{-i}} . \\
\text { Тогда } \\
d_{2 N}^{2 s, 2 N-2 s}(k, a, k-a ; q, z)=P_{q, z} \cdot \Phi(s) \Psi(s+1, N+s) \Phi^{\vee}(N-s), \\
d_{2 N}^{2 s+1,2 N-2 s-1}(k, a, k-a ; q, z)=P_{q, z} \cdot \Phi(s) \Psi(s+1, N+s+1) \Phi^{\vee}(N-s-1),
\end{gathered}
$$

где $P_{q, z}-$ числитель этих дробей, не зависящий от $s$. Легко доказать по индукции, что при $s<N$

$$
\begin{aligned}
& \sum_{m=0}^{2 s}(-1)^{m} d_{2 N}^{m, 2 N-m}(k, a, k-a ; q, z)=\frac{P_{q, z}}{1-q^{-N}} \Phi(s) \Psi(s+1, N+s) \Phi^{\vee}(N-s-1), \\
& \sum_{m=0}^{2 s+1}(-1)^{m} d_{2 N}^{m, 2 N-m}(k, a, k-a ; q, z)=-\frac{P_{q, z}}{1-q^{N}} \Phi(s) \Psi(s+2, N+s+1) \Phi^{\vee}(N-s-1) .
\end{aligned}
$$

Используя вторую формулу для $s=N-1$, получаем утверждение леммы.

Докажем утверждения теоремы 3 . Для $N=2$ они поддаются прямой проверке. Именно в этот момент происходит разделение на два случая в зависимости от $l$ и $r$. Предположим, что все утверждения теоремы верны для $N-1$, и докажем их для $N$.

Пусть $N$ нечетно. По леммам 2 и $3 d_{N}^{e}(k, l, 0 ; q, z)=d_{N-1}^{e}(k, l, k ; q, z)$. Поскольку $D_{N}(k, l, 0) \cong D_{N-1}(k, l, k)$, из предположения индукции следует, что $d_{N}(k, l, 0 ; q, z)=d_{N}^{e}(k, l, 0 ; q, z)$. Индукция по $r$, основанная на лемме 2 и следствии 1 , доказывает, что $d_{N}(k, l, r ; q, z)=d_{N}^{e}(k, l, r ; q, z)$ при $r \leqslant l$, т. е. утверждение (ii).

Утверждение (i) выводится из (ii) применением преобразования пространства $D_{N}(k, l, r)$, переводящего $e_{i}$ в $e_{N-i+1}$. 
Теперь пусть $N$ четно. Утверждение (iii) доказывается аналогично (ii). Для $r=k-l$ утверждение (iv) следует из утверждения (iii) и леммы 4 , после чего утверждение (iv) для произвольного $r \geqslant k-l$ доказывается аналогичной индукцией по $r$.

8. Чтобы получить правую часть формулы (2), рассмотрим частный случай $l=r=k$. В этом случае вершины $M_{2 m, 2 n}, M_{2 m-1,2 n}, M_{2 m, 2 n-1}$ и $M_{2 m-1,2 n-1}$ склеиваются в одну (обозначим ее через $M_{m, n}^{\prime}$ ) и, кроме того, при нечетном $N$ склеиваются между собой все вершины вида $M_{n, N-n}$ (обозначим соответствующую вершину через $M^{\prime}$ ).

Положим

$$
d_{M_{m, n}^{\prime}}=\sum_{i, j=0,1} d_{N}^{2 m-i, 2 n-j}(k, k, k ; q, z),
$$

считая, что $d_{N}^{i, j}(k, k, k ; q, z)=0$ при $i<0$ или $j<0$. Теперь правая часть формулы (2) получается из следующего утверждения.

ПРЕДЛОЖЕНИЕ 4.

$$
d_{M_{m, n}^{\prime}}^{\prime}=\frac{z^{k(m+n)} q^{k\left(m^{2}+(N+1) n-n^{2}\right)}}{\prod_{i=1}^{m}\left(1-q^{i}\right)\left(1-q^{-i-m+1} z^{-1}\right) \prod_{i=2 m+1}^{N-2 n}\left(1-q^{i} z\right) \prod_{i=1}^{n}\left(1-q^{-i}\right)\left(1-q^{-N+i+n-2} z^{-1}\right)} .
$$

Кроме того, пусть

$$
d_{M^{\prime}}=\sum_{i+j=N, i \text { четно }} d_{N}^{i, j}(k, k, k ; q, z)
$$

для нечетного $N$. Мы предлагаем следующее утверждение, характеризующее вклад особой точки многообразия $\mathscr{M}_{N}$.

ГипотезА 1. Пусть $N$ нечетно. Тогда

где $P_{N}(q, z)-$ полином.

$$
d_{M^{\prime}}=\frac{P_{N}(q, z)}{\prod_{i=1}^{N}\left(1-q^{-i} z^{-1}\right)}
$$

\section{ЛИТЕРАТУРА}

1. Feigin B., Frenkel E. Coinvariants of nilpotent subalgebras of the Virasoro algebra and partition identities. Adv. Sov. Math., Vol. 16, 1993, pp. 139-148 (hep-th/9301039).

2. Feigin B., Stoyanousky A. Quasi-particles models for the representations of Lie algebras and geometry of flag manifold, hep-th/9308079, RIMS 942; Стояновский А. В., Фейгин Б. Л. Функциональные модели представлений алгебр токов и полубесконечные клетки Шуберта. Функц. анализ и его прил., 28, вып. 1, 68-90 (1994).

3. Kedem R., Klassen T., McCoy B., Melzer E. Fermionic sum representations for conformal field theory characters. Phys. Lett. B, 307, 68-76 (1993).

4. Пухликов A. В., Хованский $A$. $Г$. Теорема Римана-Роха для интегралов и сумм квазиполиномов по виртуальным многогранникам. Алгебра и анализ, 4, 188-216 (1992).

5. Schilling A., Warnaar S. O. Supernomial coefficients, polynomial identities and $q$-series. q-alg/9701007, Ramanujan J., 2, 459-494 (1998).

6. Warnaar S. O. The Andrews-Gordon identities and $q$-multinomial coefficients. q-alg/ 9601012, Comm. Math. Phys., 184, 203-232 (1997). 